05

\title{
Влияние $\Delta E$-эффекта на полевую зависимость магнитоэлектрического эффекта в области электромеханического резонанса
}

\author{
(C) В.М. Лалетин, ${ }^{1}$ Д.А. Филиппов ${ }^{2}$ \\ ${ }^{1}$ Институт технической акустики НАН Беларуси, \\ 210023 Витебск, Беларусь \\ ${ }^{2}$ Новгородский государственный университет им. Ярослава Мудрого, \\ 173003 Великий Новгород, Россия \\ e-mail: Dmitry.Filippov@novsu.ru
}

(Поступило в Редакцию 17 мая 2017 г.)

Экспериментально исследовано влияние $\Delta E$-эффекта на полевую зависимость резонансного магнитоэлектрического эффекта в объемных композиционных материалах состава феррит-никелевая шпинель цирконат-титанат свинца в области электромеханического резонанса. Показано, что полевая зависимость величины эффекта определяется не только зависимостью пьезомагнитного коэффициента от магнитного поля, но также и зависимостью модуля Юнга. Представлена зависимость резонансной частоты эффекта от величины поля подмагничивания.

DOI: 10.21883/JTF.2018.02.45406.2337

\section{Введение}

Магнитоэлектрический (МЭ) эффект, открытый более полувека назад, в последнее время привлекает к себе все большее внимание исследователей, о чем говорит рост публикаций на эту тему [1]. В последнее время синтезировано большое количество новых материалов, получивших общее название „мультиферроики“, в которых наблюдаются эффекты, связывающие между собой магнитные и электрические характеристики материала. К таким материалам относятся и композиционные МЭ материалы, созданные на основе магнитострикционной и пьезоэлектрической фаз. МЭ эффект является новым свойством таких материалов и относится к классу вторичных эффектов или так называемых „product

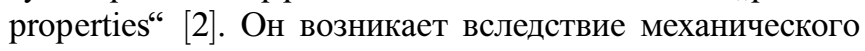
взаимодействия магнитострикционной и пьезоэлектрической подсистем. При помещении образца в переменное магнитное поле в магнитострикционной компоненте возникают механические напряжения, которые посредством механического взаимодействия передаются в пьезоэлектрическую фазу, вследствие чего происходит изменение поляризации, что приводит к возникновению электрического напряжения. Поскольку МЭ эффект в композитах связан с механическими колебаниями, то в области электромеханического резонанса величина МЭ эффекта резко возрастает [3,4]. Вследствие того, что магнитострикция является нелинейным по намагниченности (магнитному полю) эффектом, то очевидно, что линейный по переменному магнитному полю эффект может наблюдаться только при наличии поля подмагничивания. Зависимость величины эффекта от поля подмагничивания получила название ,полевая зависимость“ МЭ эффекта. Эта зависимость имеет максимум [5], наличие которого связывают с зависимостью пьезомагнитного коэффициента $q$ от магнитного поля. Пьезомагнитный коэффициент $q$ определяется как

$$
q=\left.\frac{\partial \lambda}{\partial H}\right|_{H=H_{\text {bias }}},
$$

где $\lambda$ - магнитострикция, $H_{\text {bias }}$ - поле подмагничивания. Несмотря на то что основной вклад в зависимость величины эффекта от магнитного поля дает пьезомагнитный коэффициент, на величину эффекта, особенно в области электромеханического резонанса, оказывает и зависимость модуля Юнга от магнитного поля или так называемый $\Delta E$-эффект. В настоящей работе проанализировано влияние этого явления на величину МЭ эффекта в области электромеханического резонанса.

\section{Материалы и методы исследования}

Для проведения исследований использовался объемный композиционный материал, полученный путем спекания порошков феррита и пьезоэлектрика. С этой целью были приготовлены образцы, состоящие из смеси с массовым содержанием 50\% порошка ЦТС 23 и 50\% порошка модифицированного феррита никеля $\mathrm{NiFe}_{1.9} \mathrm{Co}_{0.02} \mathrm{O}_{4}$ с добавлением в него окиси титана с массовым содержанием $1.5 \%$. Образцы спекали в тигле со свинецсодержащей засыпкой в течение двух часов при температуре $1240^{\circ} \mathrm{C}$. Скорость охлаждения образцов не превышала $50 \mathrm{deg}$ в $1 \mathrm{~h}$. Электроды наносили путем вжигания серебряной пасты при температуре $650^{\circ} \mathrm{C}$ в течение $20 \mathrm{~min}$. Образцы имели форму дисков диаметром $8.7-8.8 \mathrm{~mm}$ и толщиной $0.8-0.9 \mathrm{~mm}$. Поляризацию материалов осуществляли при температуре $80^{\circ} \mathrm{C}$ в течение $2 \mathrm{~h}$ в электрическом поле $4 \mathrm{kV} / \mathrm{mm}$ с последующим 
охлаждением в этом поле до комнатной температуры в течение $0.5 \mathrm{~h}$.

Для исследования резонансного МЭ эффекта использовался метод, основанный на регистрации переменного напряжения, возникающего на электродах образца при помещении его в переменное и постоянное магнитные поля. Переменное магнитное поле создавалось катушками Гельмгольца и его величина в эксперименте была равна $h=0.8 \mathrm{~A} / \mathrm{m}$. Полевая зависимость эффекта исследовалась путем изменения поля подмагничивания, которое получали электромагнитом. В эксперименте исследовался поперечный МЭ эффект, при котором переменное и постоянное магнитные поля лежали в плоскости образца, и были направлены перпендикулярно вектору поляризации. Выбор поперечного эффекта был обусловлен тем, что величина резонансного МЭ эффекта при поперечной ориентации полей в несколько раз превосходит его значение при продольной ориентации полей.

МЭ коэффициент по напряжению, являющийся главной характеристикой МЭ эффекта, рассчитывался по формуле

$$
\alpha_{E}=u /(h t),
$$

где $u$ - напряжение, возникающее на электродах образца, $h$ - напряженность переменного магнитного поля, $t$ - толщина образца.

\section{Результаты исследования и их обсуждение}

Исследования резонансного МЭ эффекта производят, как правило, следующим образом: на низкой частоте $(f=1 \mathrm{kHz})$ при неизменном значении переменного магнитного поля $h$ определяют поле подмагничивания $H_{m \text {, bias, }}$ соответствующее максимальному значению эффекта, а затем при данном значении поля снимают частотную зависимость эффекта. Резонансную частоту, соответствующую полю подмагничивания $H_{m \text {, bias }}$, будем называть основной резонансной частотой $f_{0 \text {,res. }}$ В дан-

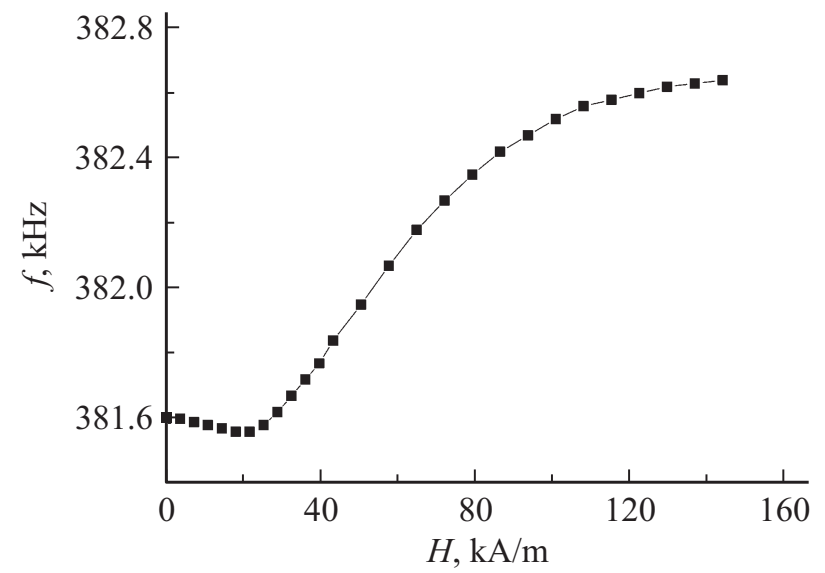

Рис. 1. Полевая зависимость резонансной частоты.

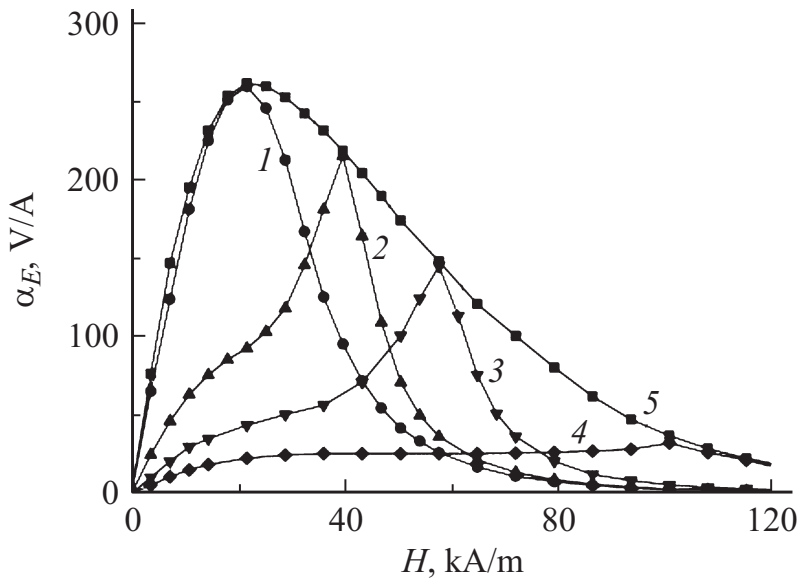

Рис. 2. Полевые зависимости МЭ коэффициента, полученные при частотах, $f$, kHz: 1 - 381.56, 2 - 381.76, 3 - 382.06, 4 $382.56,5$ - в режиме непрерывной настройки на резонансную частоту.

ном случае $H_{m \text {, bias }}=22 \mathrm{kA} / \mathrm{m}$, а значение основной резонансной частоты было равно $f_{0 \text {,res }}=381.56 \mathrm{kHz}$. Как известно [4], частота электромеханического резонанса определяется геометрическими размерами образца (радиусом диска), плотностью материала и его упругими свойствами (модулем Юнга). При изменении поля подмагничивания происходит изменение в магнитострикционной фазе модуля Юнга, вследствие чего происходит изменение частоты электромеханического резонанса. На рис. 1 представлена зависимость резонансной частоты от поля подмагничивания. Как видно из рисунка, в полях до $27 \mathrm{kA} / \mathrm{m}$ наблюдается небольшой отрицательный $\Delta E$-эффект, вследствие чего величина резонансной частоты уменьшается при увеличении магнитного поля. Минимум резонансной частоты совпадает с основной резонансной частотой $f_{0 \text {,res }}$ и наблюдается в магнитном поле $H_{m \text {, bias }}=22 \mathrm{kA} / \mathrm{m}$. При дальнейшем увеличении магнитного поля резонансная частота растет и выходит на насыщение при магнитном поле свыше $140 \mathrm{kA} / \mathrm{m}$. Это область положительного $\Delta E$-эффекта.

Исследование поведения МЭ коэффициента по напряжению в области электромеханического резонанса показали, что полученные результаты можно разбить на две группы - полевые зависимости на частотах выше и ниже основной резонансной частоты.

Результаты исследований полевых зависимостей МЭ коэффициента на частотах больше основной резонансной частоты представлены на рис. 2. На рис. 3 представлены полевые зависимости, полученные на частотах ниже основной резонансной частоты.

Как видно из рисунков, все зависимости имеют четко выраженный максимум, который в первом случае имеет острый пик и с ростом частоты измерения смещается в сторону увеличения поля подмагничивания, а во втором случае имеет типичную зависимость, свойственную композиционным материалам, и наблюдается при одном и том же значении поля подмагничивания. Интересный 


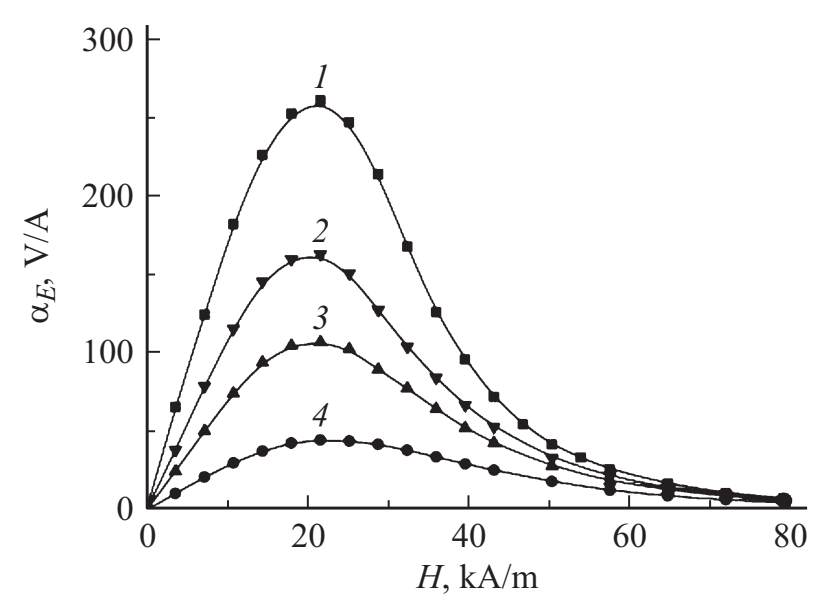

Рис. 3. Полевые зависимости МЭ коэффициента, полученные при частотах, $f$, kHz: $1-381.56,2-381.46,3-381.36$, $4-381.06$.

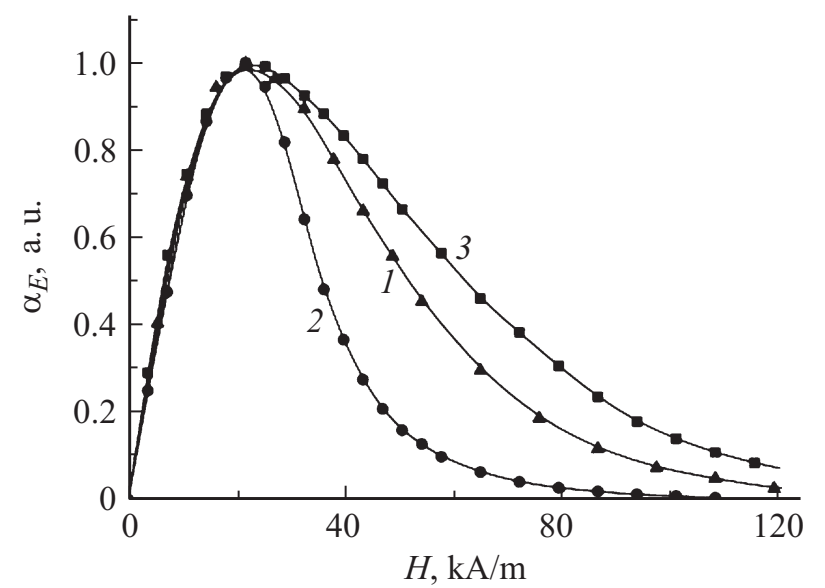

Рис. 4. Полевые зависимости МЭ коэффициента, полученные 1 - при низкой частоте $f=1 \mathrm{kHz}, 2-$ при основной резонансной частоте $f=381.56 \mathrm{kHz}, 3-$ в режиме непрерывной настройки на резонансную частоту.

результат получен при измерении полевой зависимости на частоте $382.56 \mathrm{kHz}$. В интервале значений магнитного поля от 36 до $80 \mathrm{kA} / \mathrm{m}$ величина МЭ сигнала практически не меняется.

При исследовании полевой зависимости МЭ коэффициента на частотах, превышающих основную резонансную частоту $(381.76,382.06,382.56 \mathrm{kHz})$, значения их подмагничивающих полей попадают в область полевой зависимости резонансной частоты. Это приводит к возникновению пика на полевой зависимости, величина которого будет определяться полевой зависимостью резонансного МЭ коэффициента, полученного в режиме непрерывной настройки на резонансную частоту (рис. 2, кривая 5). Отсутствие пика на полевой зависимости МЭ коэффициента, полученного на основной резонансной частоте, объясняется тем, что основная резонансная частота находится в точке перегиба кривой зависимости резонансной частоты от магнитного поля (рис. 4). Наличие плато в области магнитного поля от 36 до $80 \mathrm{kA} / \mathrm{m}$ (рис. 2, кривая 4), по-видимому, объясняется наложением ряда эффектов: зависимостью пьезомагнитного коэффициента и МЭ резонансного коэффициента от магнитного поля, $\Delta E$-эффектом.

Как уже отмечалось, полевые зависимости МЭ коэффициента, полученные на частотах, меньше основной резонансной частоты, имеют типичный вид, свойственный композиционным материалам. Это вызвано тем, что данные частоты не попадают в область $\Delta E$-эффекта и в этом случае происходит резонансное усиление сигнала, величина которого будет зависеть от рассогласования резонансной частоты и добротности резонатора.

На рис. 4 представлены полевые зависимости низкочастотного $(f=1 \mathrm{kHz})$ МЭ коэффициента и полевые зависимости, полученные на частоте основного резонанса и в режиме непрерывной настройки на резонансную частоту. Для удобства сравнения значения МЭ коэффициентов выражены в относительных единицах $\alpha_{E \text {, a.u. }}=\frac{\alpha_{E}(H)}{\alpha_{E, \max }}$. Из полученных результатов следует, что полевые зависимости МЭ коэффициента имеют типичный вид, свойственный композиционным материалам, во всех случаях максимум сигнала наблюдается при значении подмагничивающего поля, равного $22 \mathrm{kA} / \mathrm{m}$. На начальном участке полевой зависимости от 0 до $22 \mathrm{kA} / \mathrm{m}$ кривые практически совпадают. Однако в магнитных полях свыше $22 \mathrm{kA} / \mathrm{m}$ наблюдаются значительные расхождения. Полевая зависимость МЭ коэффициента, полученная в режиме непрерывной настройки на резонансную частоту, более растянута по магнитному полю, чем полевая зависимость низкочастотного МЭ коэффициента. И наоборот, полевая зависимость МЭ коэффициента, полученная на основной резонансной частоте, сжата по сравнению с низкочастотной зависимостью. Это объясняется тем, что на низких частотах полевая зависимость МЭ коэффициента определяется зависимостью пьезомагнитного коэффициента. В области электромеханического резонанса величина МЭ сигнала определяется, помимо этого, добротностью образца, $\Delta E$-эффектом и условиями эксперимента. При измерении полевой зависимости МЭ коэффициента на основной резонансной частоте в магнитных полях свыше $22 \mathrm{kA} / \mathrm{m}$ наблюдается уход резонансной частоты, что приводит к рассогласованию резонанса и падению МЭ сигнала. И наоборот, измерение полевой зависимости МЭ коэффициента в режиме непрерывной настройки на резонансную частоту позволяет получить максимальный МЭ сигнал, что приводит к расширению полевой зависимости МЭ коэффициента.

\section{Выводы}

Исследована полевая зависимость МЭ коэффициента в области электромеханического резонанса. Показано, что наличие $\Delta E$-эффекта приводит к зависимости резонансной частоты от напряженности магнитного поля. 
В области полей, меньших значения поля, соответствующего максимуму МЭ эффекта, резонансная частота уменьшается. При дальнейшем увеличении поля резонансная частота растет и выходит на насыщение.

Полевые зависимости МЭ коэффициента на частотах вблизи основной резонансной частоты при значениях больше или меньше ее имеют различный характер. Все зависимости имеют четко выраженный максимум, который в первом случае имеет острый пик и с ростом частоты измерения смещается в сторону увеличения поля подмагничивания. Во втором случае зависимость имеет типичный вид свойственный композиционным материалам и наблюдается при одном и том же значении поля подмагничивания. Это обусловлено влиянием $\Delta E$ эффекта на резонансную частоту.

Работа выполнена при поддержке гранта совместного конкурса РФФИ - БРФФИ: Российский проект № 1652-00184 и Белорусский проект № Ф16Р-130.

\section{Список литературы}

[1] Звездин А.К., Пятаков А.П .// УФН. 2012. Т. 182. № 6. C. 593-620.

[2] Van Suchtelen J. // Philips Res. Rep. 1972. Vol. 27. P. $28-37$.

[3] Филиппов Д.А., Бичурин М.И., Петров В.М., Лалетин В.М., Поддубная H.H., Srinivasan G. // Письма в ЖТФ. 2004. Т. 30. Вып. 1. С. 15-20.

[4] Филиппов Д.А., Бичурин М.И., Петров В.М., Лалетин В.М., Srinivasan G. // ФTT. 2004. Vol. 46. C. 1621-1627.

[5] Филиппов Д.А., Лалетин В.М., Srinivasan G. // ЖТФ. 2012. Т. 82. Вып. 1. С. 47-51. 